\title{
Triggering dynamic structural changes in lipid membranes
}

Nicholas Jan Brooks ${ }^{1}$, Nicola McCarthy ${ }^{1}$, Arwen Tyler ${ }^{1}$, Hanna Barriga ${ }^{1}$, Sowmya Purushothaman ${ }^{1}$

${ }^{1}$ Department Of Chemistry, Imperial College London, London, United Kingdom E-mail: n.brooks@imperial.ac.uk

Lipid membrane structural dynamics and micromechanics are vitally important to a wide range of cellular processes including mediating protein activity, signaling, material transport and apoptosis (programmed cell death). Developing model systems to study the structural and energetic behavior of membranes, and methods to trigger changes in these parameters are essential to understanding the contributions of the many components that make up biological membranes.

We have recently developed a range of novel instruments for studying soft matter and biological system at non-ambient conditions and out-of-equilibrium. Amongst these, our new platforms for high pressure and pressure-jump microscopy [1], small angle X-ray diffraction (SAXS) [2][3] and spectroscopy have led to a series of exciting studies of the pressure dependence of key micromechanical membrane parameters and membrane structural behaviour.

Of particular interest are our recent first measurements of the lateral structure of membranes under pressure. This has allowed us to measure pressure induced changes in the bending rigidity, viscosity and permeability of lipid bilayers, and rapidly trigger membrane domain growth in both model lipid membranes and plasma membrane vesicles. In addition, hydrostatic pressure has played a key roll in the generation and control of highly swollen interconnected cubic lipid structures which have enormous potential for encapsulation and controlled release of bioactive molecules, and large membrane protein crystalisation.

[1] McCarthy, N. L. C., Ces, O., Law, R. V., Seddon, J. M. \& Brooks, N. J. (2015). Chem. Commun. 51, 8675-8678.

[2] Tyler, A. I. I., Barriga, H. M. G., Parsons, E. S., McCarthy, N. L. C., Ces, O., Law, R. V., Seddon, J. M. \& Brooks, N. J. (2015). Soft Matter 11, 3279-3286.

[3] Barriga, H. M. G., Law, R. V., Seddon, J. M., Ces, O. \& Brooks, N. J. (2016). PCCP, 18, 149-155.

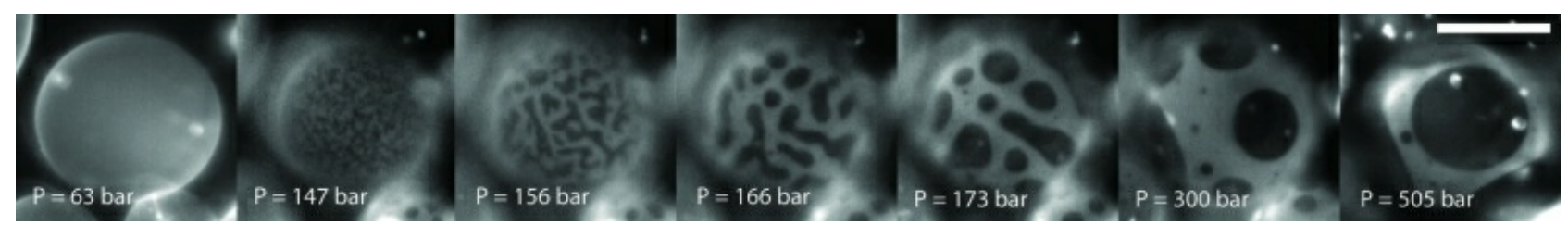

Keywords: Lipids, High pressure, Membranes 